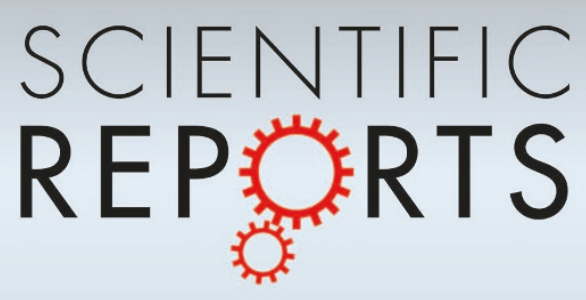

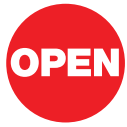

SUBJECT AREAS:

BIOINFORMATICS

EARTH AND ENVIRONMENTAL

SCIENCES

PLANT SCIENCES

BIODIVERSITY

Received

25 May 2011

Accepted

6 July 2011

Published

21 July 2011

Correspondence and requests for materials should be addressed to M.Y.S.

(mark.stoeckle@rockefeller.edu)

\title{
Commercial Teas Highlight Plant DNA Barcode Identification Successes and
} Obstacles

\author{
Mark Y. Stoeckle', Catherine C. Gamble², Rohan Kirpekar ${ }^{2}$, Grace Young ${ }^{2}$, Selena Ahmed ${ }^{3}$ \\ \& Damon P. Little ${ }^{4}$
}

'Program for the Human Environment, The Rockefeller University, New York, NY 10065, USA, ${ }^{2}$ rinity School, New York, NY 10024, USA, ${ }^{3}$ Department of Biology, Tufts University, Medford, MA 02115 , USA, ${ }^{4}$ Lewis B. and Dorothy Cullman Program for Molecular Systematics, The New York Botanical Garden, Bronx, New York, NY 10458, USA.

Appearance does not easily identify the dried plant fragments used to prepare teas to species. Here we test recovery of standard DNA barcodes for land plants from a large array of commercial tea products and analyze their performance in identifying tea constituents using existing databases. Most (90\%) of 146 tea products yielded $r b c L$ or matK barcodes using a standard protocol. Matching DNA identifications to listed ingredients was limited by incomplete databases for the two markers, shared or nearly identical barcodes among some species, and lack of standard common names for plant species. About $1 / 3$ of herbal teas generated DNA identifications not found on labels. Broad scale adoption of plant DNA barcoding may require algorithms that place search results in context of standard plant names and character-based keys for distinguishing closely-related species. Demonstrating the importance of accessible plant barcoding, our findings indicate unlisted ingredients are common in herbal teas.

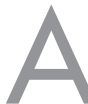

queous infusions prepared from dried plants, broadly known as teas, are popular beverages with desirable physiologic activities and potential health benefits. Accurate labeling is important for consumers, marketers, and regulators, as tea constituents cannot be easily identified to species by visual appearance. Their taxonomic diversity and fragmentary nature present a ready and demanding test of DNA-based identification. Here we report the successes with and obstacles to identifying tea ingredients using a short DNA sequence from a uniform locality within the genome, DNA barcoding ${ }^{1}$.

Tea properly refers to infusions prepared from leaves of the tea plant, Camellia sinensis (L.) Kuntze, an evergreen flowering tree in the family Theaceae, native to the mountainous regions of southwestern China and neighboring countries ${ }^{2-4}$. The two main commercial varieties are small-leafed C. sinensis var. sinensis, adapted to cool weather and high altitude, and large-leafed C. sinensis var. assamica (J. W. Mast.) Kitam., which grows well in tropical and sub-tropical environments. Tea plant leaves contain a high concentration of phytochemicals including polyphenolic catechins and the methylxanthine caffein $\mathrm{e}^{5-11}$. Tea drinking originated in southern China at least 2000 years ago, and today tea is the most widely consumed beverage in the world ${ }^{12,13}$. Different processing methods, ranging from drying and baking to months of microbial fermentation, produce the variety of tea types - white, green, black, oolong, and pu-erh - which differ in catechin content and antioxidant activity $^{14,15}$.

In addition to $C$. sinensis, infusions are prepared from a diversity of other plants and plant parts-beverages also commonly referred to as tea. In the following we use "CS" to indicate C. sinensis and "herbal" for other plants. Some herbal teas have pharmacologically active compounds and may have therapeutic or toxic effects. Fatalities and serious illnesses have occurred after drinking herbal teas, caused by overdose, mislabeled products, or allergic reactions ${ }^{16-18}$.

In 2009, the Plant Working Group of the Consortium for the Barcode of Life (CBOL) endorsed a proposal to use defined portions of the plastid genes $r b c L$ ( $\sim 550$ bp segment) and matK ( $\sim 790$ bp segment) as standard barcodes for land plants ${ }^{19}$. These and other candidate markers have been tested in various floristic and taxonomic settings $\mathrm{s}^{20-24}$. As compared to animals, plants generally have less barcode variation both within and among species. A relatively large proportion of plants $(\sim 15 \%-30 \%)$ share barcodes among multiple species. Plant barcodes generally do not exhibit the strong clustering pattern observed in most animal species (intraspecific variation « interspecific variation). These observations apply even when longer sequences or additional markers are sampled, 
which may reflect fundamental differences in plant and animal biology and evolution ${ }^{23}$. Notwithstanding these limitations, standard plant barcodes are efficacious in a number of scientific and applied settings and have enormous potential for wider use ${ }^{25}$.

In this study we explored a practical application of plant barcoding: matching commercial tea ingredients to product labels. We searched a public reference database for the closest match to each barcode sequence and compared the result to the listed ingredients. Because the tea specimens are morphologically unrecognizable, we cannot know with certainty if the source plants are represented in the reference database, a realistic and difficult test of barcode identification.

\section{Results}

Barcode recovery, haplotypes, matches. Using single sets of primers for each locus, readable $\mathrm{rbcL}$ or $\mathrm{mat} K$ barcodes were recovered from $131(90 \%)$ of 146 tea products, including $96 \%$ of CS and $84 \%$ of herbal teas. $r b c L$ was recovered from $113 / 146$ (77\%), matK from $108 / 136(79 \%)$, and both from 90/136 (66\%). A total of 253 readable sequences were obtained, comprising $48 \mathrm{rbcL}$ and 40 matK haplotypes (Figs. 1,2; additional details in Supplementary Tables S1,S2 online). There were no insertions or deletions in $r b c L$ sequences; the matK alignment contained 14 different types of insertions or deletions. For each haplotype, BLAST searches of
GenBank and Barcode of Life databases were performed. The closest match in each database was recorded. As compared to results with GenBank, BOLD matches were on average lower identity and fewer were label ingredients, indicating that at the time of the study BOLD was less well populated with barcodes of plants used in commercial tea products. As a result, subsequent analyses were performed using GenBank. The $r b c L$ haplotypes matched 42 species in 24 families; the matK haplotypes matched 25 species in 16 families (Figs. 1,2).

Taking into account uncertainties arising from incomplete databases, shared barcodes, and ambiguous common names, of $48 \mathrm{rbcL}$ haplotypes, 32 were assigned to species, 10 to genus, and 6 to family. Of 40 mat $K$ haplotypes, 27 were assigned to species, 8 to genus, and 5 to family (Figs. 1,2). In most cases $(58 \%)$, barcodes recovered from commercial tea products matched listed ingredients. It should be noted that our study was designed to enable comparison between CS and herbal teas, and not among individual products or manufacturers. Given this and potential liability issues, we assigned arbitrary alphanumeric codes to each product to protect the manufacturer's identity. Most of the barcodes that did not match listed ingredients reflected an incomplete reference database, lacking either a record for the relevant species or a record of an intraspecific variant. For example, an herbal tea labeled "Marshmallow (Althaea officinalis)" produced an $r b c L$ sequence closest to Anisodontea

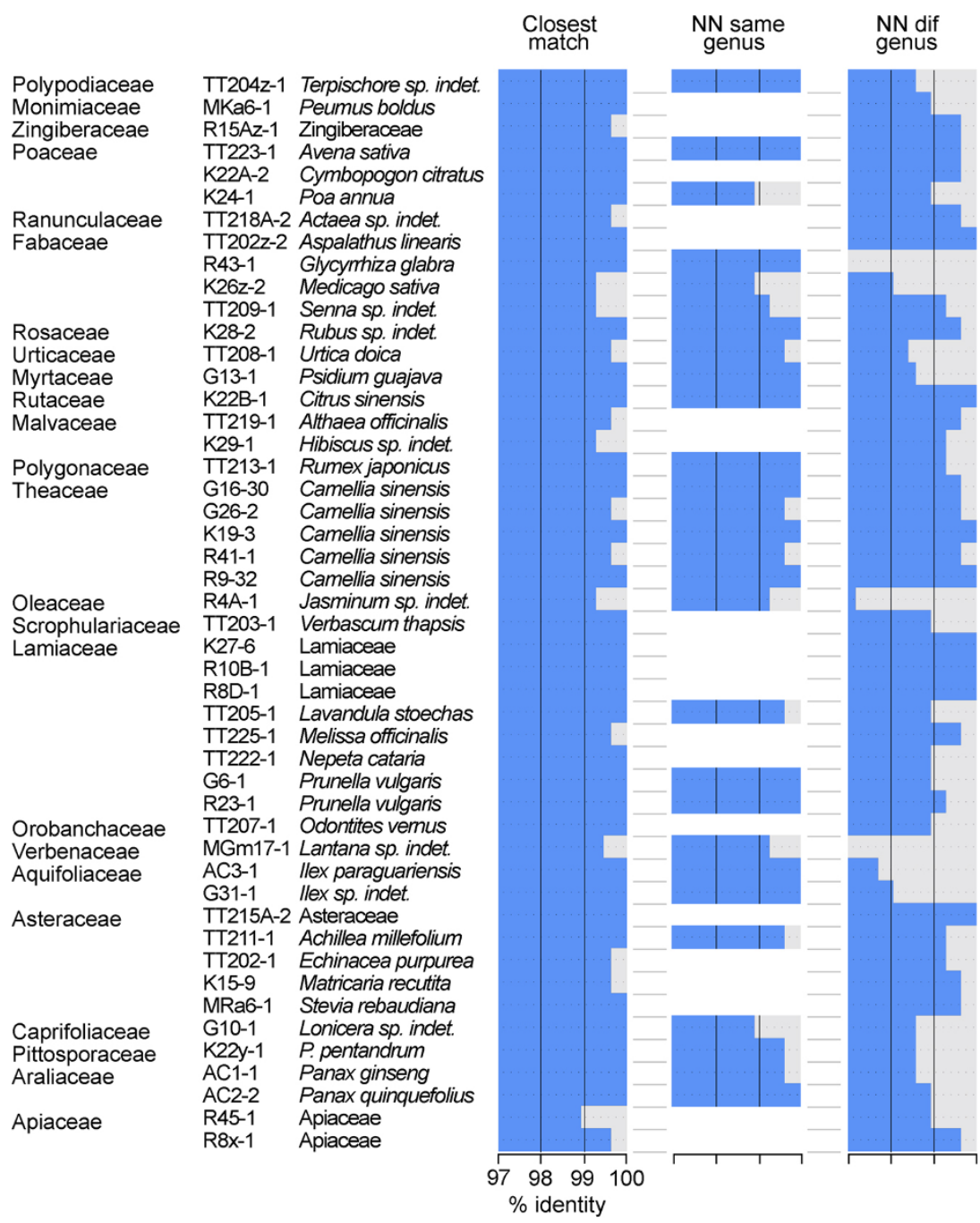

Figure $1 \mid r b c L$ barcode identifications. For each haplotype, alphanumeric code, number of isolates, identification, and graphic representation of match results are shown. Color bars depict percent identity of closest match, nearest neighbor (NN) in the same genus, and NN in a different genus, with scale at bottom. Haplotypes for which the second closest match was in a different genus have a blank in "NN same genus" column.

(Note: $P$. pentandrum $=$ Pittosporum pentandrum). 


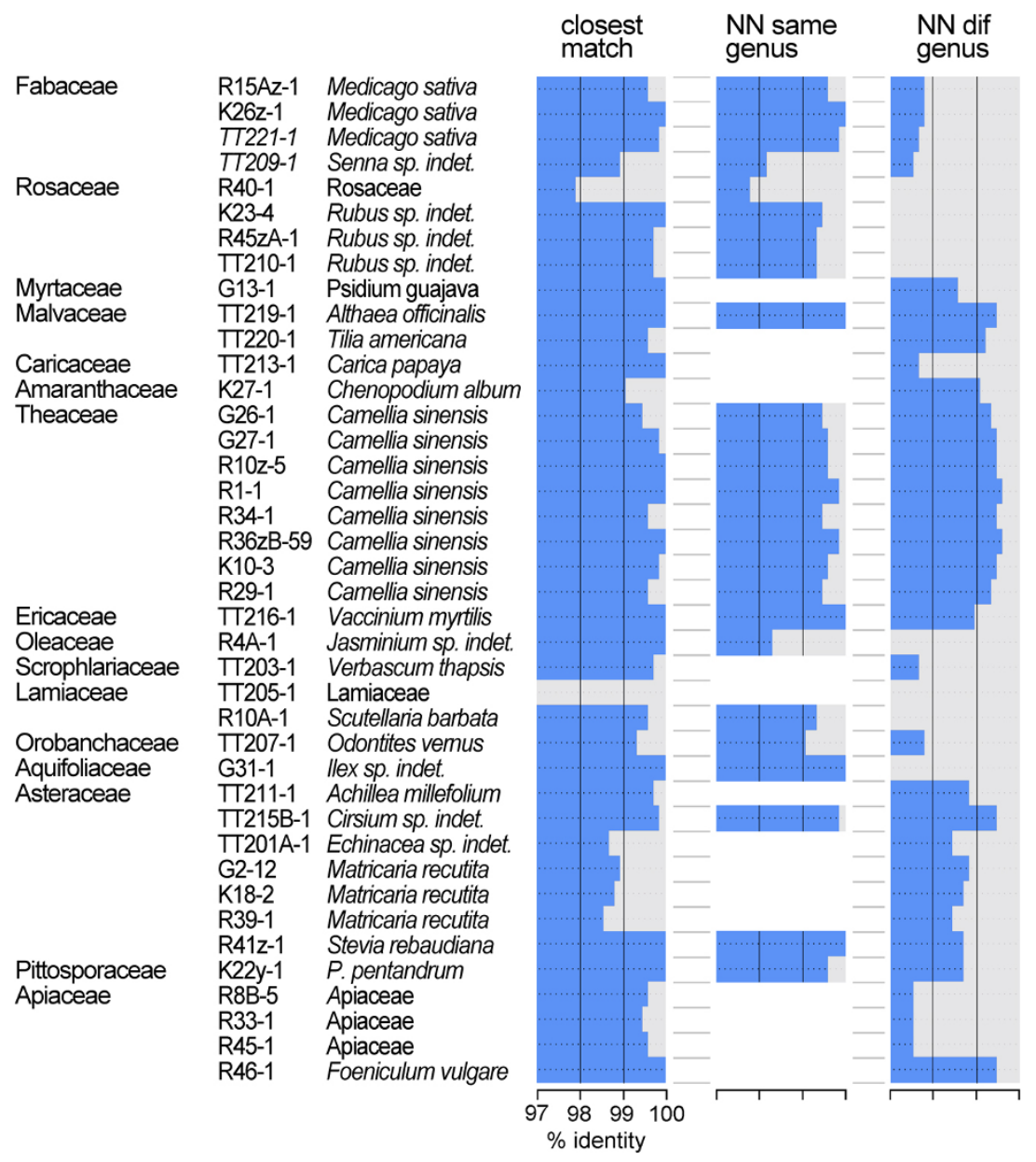

Figure $2 \mid$ matKbarcode identifications. For each haplotype, alphanumeric code, number of isolates, identification, and graphic representation of match are shown as described in Fig. 1 legend.

triloba (1 mismatch, 99.8\% identity). However, at the time of the study there were no GenBank $r b c L$ records for $A$. officinalis. Overall, at the time of the study about one-third of plant species listed on product labels lacked $r b c L$ or matK records in GenBank. Reflecting incomplete representation of intraspecific variants, more than half of $C$. sinensis tea products yielded an $r b c L$ barcode $100 \%$ identical to congeneric species C. oleifera and C. sasanqua but with one mismatch compared to the $C$. sinensis $r b c L$ record.

Barcode identifications were incompatible with listed ingredients for some products, including 21/60 (35\%) herbal and 3/70 (4\%) CS teas (Table 1). Some of the non-label DNAs matched plants used in other tea products, some matched common weeds or other non-food plants, and some could not be identified. The most common non-label ingredient, found in seven products, was chamomile (Matricaria recutita). Four herbal teas yielded sequences identified as tea plant (C. sinensis), although none listed ingredients in the tea family (Theaceae). Regarding non-food plants, a product labeled "St. John's wort (Hypericum perforatum)," a flowering plant, yielded an $r b c L$ sequence identical to that of several fern species. A barcode from an herbal tea matched Poa annua, a widely cultivated meadow grass. Four products yielded barcodes closely matching plants in Apiaceae, the parsley family, although the particular species could not be determined. Apiaceae includes many food plants and ubiquitous wild relatives, but for the products in question none of the listed ingredients were in this family.

Taxonomic resolution. For most $r b c L$ haplotypes, the differences between closest match, nearest neighbor (NN) in the same genus, and NN in a different genus were modest or absent. Among the 48 haplotypes, the average percent identity was $99.9 \%$ for closest, $99.8 \%$ for congeneric $\mathrm{NN}$, and $99.2 \%$ for $\mathrm{NN}$ in a different genus, or about $0.6,1.1$, and 4.6 nucleotide differences respectively (Fig. 1; additional details in Supplementary Table S1 online). Of $32 \mathrm{rbcL}$ haplotypes with $100 \%$ match, 15 were also identical to one or more congeneric species and eight were identical to one or more species in a different genus.

For $\operatorname{mat} K$, the average identities were $99.5 \%$ for closest match, $99.5 \%$ for $\mathrm{NN}$ congeneric, and $98.1 \%$ for $\mathrm{NN}$ different genus, or about 3.8, 3.8, and 14.3 nucleotide differences (Fig. 2; additional details in Supplementary Table S2 online). Of 14 haplotypes with a $100 \%$ match, three were also identical to one or more congeneric species, and none were identical to species in a different genus.

C. sinensis $r b c L$ nucleotide sequence polymorphism. We observed nucleotide variation (A or C) in CS $r b c L$ sequences at a site corresponding to position 68 of the coding region (gi 7525012:54958-56397 was used as a reference), with the predicted predicted amino acid being either asparagine (68A) or threonine (68C). The 68A sequence was identical to the $C$. sinensis $r b c L$ GenBank record, whereas the $68 \mathrm{C}$ variant was identical to $r b c L$ sequences of several congeneric species (C. albogigas, C. granthamiana, C. japonica, C. oleifera, C. sasanqua) and a related species Tutcheria hirta. Among tea products for which geographic or tea type information was available, the $68 \mathrm{C}$ variant was associated with products from India as compared to China ( $94 \%$ vs. $31 \%, p<0.0001)$ and with black vs. green tea $(93 \%$ vs. $19 \%, \mathrm{p}<0.0001)$. Among vouchered specimens, the $68 \mathrm{C}$ variant was strongly associated with $C$. sinensis 
Table 1 | DNA barcode identification of unlisted ingredients.

Product:

Label:

Non-label DNA:

Comment:

Product:

Label:

Non-label DNA:

Comment:

Product:

Label:

Non-label DNA:

Comment:

Product:

Label:

Non-label DNA:

Comment:

Product:

Label:

Non-label DNA:

Comment:

Product:

Label:

Non-label DNA:

Comment:

Product:

Label:

Non-label DNA:

Comment:

Product:

Label:

Non-label DNA:

Comment

Product:

Label:

Non-label DNA:

Comment:

Product:

Label:

Non-label DNA:

Comment:

Product:

Label:

Non-label DNA:

Comment:

Product:

Label:

Non-label DNA:

Comment:
G1

apple pieces, vitamin C, citric acid, natural flavor

tea (Camellia sinensis)

G1 matK 100\% identity to Camellia sinensis, familiy Theaceae. No listed ingredients in Theaceae.

G2

apple pieces, orange peel, rosehips, hibiscus, cornflower blossoms, clove, cinnamon, anise, pepper, natural flavor

chamomile (Matricaria recutita)

G2 rbcL 99.6\% identity Pentzia incana, G2 matK 98.9\% match Achillea millefolium, both family Asteraceae. rbcL and matK sequences most likely represent chamomile (Matricaria recutita), based on 100\% match to partial M. recutita sequences in GenBank and recovery of identical or nearly identical sequences from products listing chamomile as sole ingredient. Listed ingredient cornflower is not an approved name for chamomile ${ }^{35}$. It refers to Centaurea cyanus, family Asteraceae. Compared to closest match, G2 sequence is more distant from C. cyanus rbcL (96.8\%) (AB530955); no C. cyanus matK sequences in GenBank. Other ingredients in different families.

G4

raspberry pieces, apple pieces, orange peel, rosehips, hibiscus, lemongrass, vitamin C, natural raspberry flavor chamomile (Matricaria recutita)

G4 matK 98.9\% match Achillea millefolium, family Asteraceae, most likely representes chamomile (Matricaria recutita) (see Comment under G2). No listed ingredients in Asteraceae.

G6

Prunella vulgaris

chamomile (Matricaria recutita)

G6 matK 98.9\% match Achillea millefolium, family Asteraceae, most likely represents chamomile (Matricaria recutita) (see

Comment under G2). No listed ingredients in Asteraceae.

G10

honeysuckle flower

chamomile (Matricaria recutita)

G10 matK 98.9\% identity Achillea millefolium, family Asteraceae, most likely represents chamomile (Matricaria recutita) (see Comment under G2). No listed ingredients in Asteraceae.

K21

pau d'arco inner bark

tea (Camillia sinensis)

K2 1 rbcL and matK 100\% match Camellia sinensis, family Theaceae. No listed ingredients in Theaceae.

K22

rosehips, orange peel, chamomile flowers, lemongrass, lemon myrtle, hibiscus flowers, nana mint, natural citrus flavors and other natural flavors

Taiwanese cheesewood (Pittosporum pentandrum)

K22 rbcL and matK 100\% match Pittosporum pentandrum (Taiwanese cheesewood), family Pittosporaceae. No listed ingredients in Pittosporaceae.

K24

ginger root, natural flavors, linden, lemon peel, blackberry leaves, lemongrass, citric acid annual bluegrass (Poa annua)

K24 rbcL 100\% match to Poa annua (annual bluegrass), family Poaceae. Listed ingredient lemongrass, Citropogon citratus, is also in Poaceae. However, compared to closest match, K24 sequence is more distant from C. citratus (94.0\%) (GQ436383). No other ingredients in Poaceae.

K27

eleuthero, peppermint, cinnamon, ginger, chamomile, west indian lemongrass, licorice, catnip, tilia flowers, natural lemon flavor, hops, vitamins B6 and B12

white goosefoot (Chenopodium album)

In GenBank, K27 matK 99.2\% match Rhagodia baccata and Chenopodium album, both family Amaranthaceae. In BOLD, K27 matK is 100\% match to Chenopodium album. No listed ingredients in Amaranthaceae.

R8

mate, licorice, rosehips, mint, pineapple chunks, natural flavors

tea (Camellia sinensis), parsley family (Apiaceae)

R8 rbcL1 100\% match to Camellia oleifera, family Theaceae. No listed ingredients in Theaeceae. R8 rbcL2 99.8\% and matK 99.7\% match Pimpinella saxifraga, family Apiaceae. No listed ingredients in Apiaceae.

R 10

tea, lemongrass, lemon verbena, spearmint, natural flavors

chamomile (Matricaria recutita), skullcap (Scutellaria barbata)

R10 rbcL 99.6\% match Pentzia incana, R 10 matK1 98.9\% match Achillea millefolium, both family Asteraceae, likely represents chamomile (Matricaria recutita) (see Comment under G2). No listed Ingredients in Asteraceae. R10 matK2 99.7\% match Scutellaria barbata, family Lamiaceae. Listed Ingredient spearmint (Mentha spicata) is also in Lamiaceae. However compared to closest match, R 10 matK2 is relatively distant from M. spicata (91.8\%) (GU381684). No other ingredients in Lamiaceae.

R 15

black tea plus rooibos, black pepper, cardamom, cinnamon, ginger, organic cane sugar, natural flavors

chamomile (Matricaria recutita), alfalfa (Medicago sativa)

R 15 rbcL 99.6\% match Pentzia incana and R 15 matK1 98.9\% match Achillea millefolium, both family Asteraceae, likely represents chamomile (Matricaria recutita) (see Comment under G2). No ingredients in Asteraceae. R15 matK2 99.7\% match Medicago sativa, family Fabaceae. Listed ingredient rooibos (Aspalathus linearis) is also family Fabaceae. No A. linearis matK sequences in GenBank for comparison, but $A$. linearis and $M$. sativa $r b c L$ sequences show limited identity $(93.6 \%)$. Other ingredients in different families. 


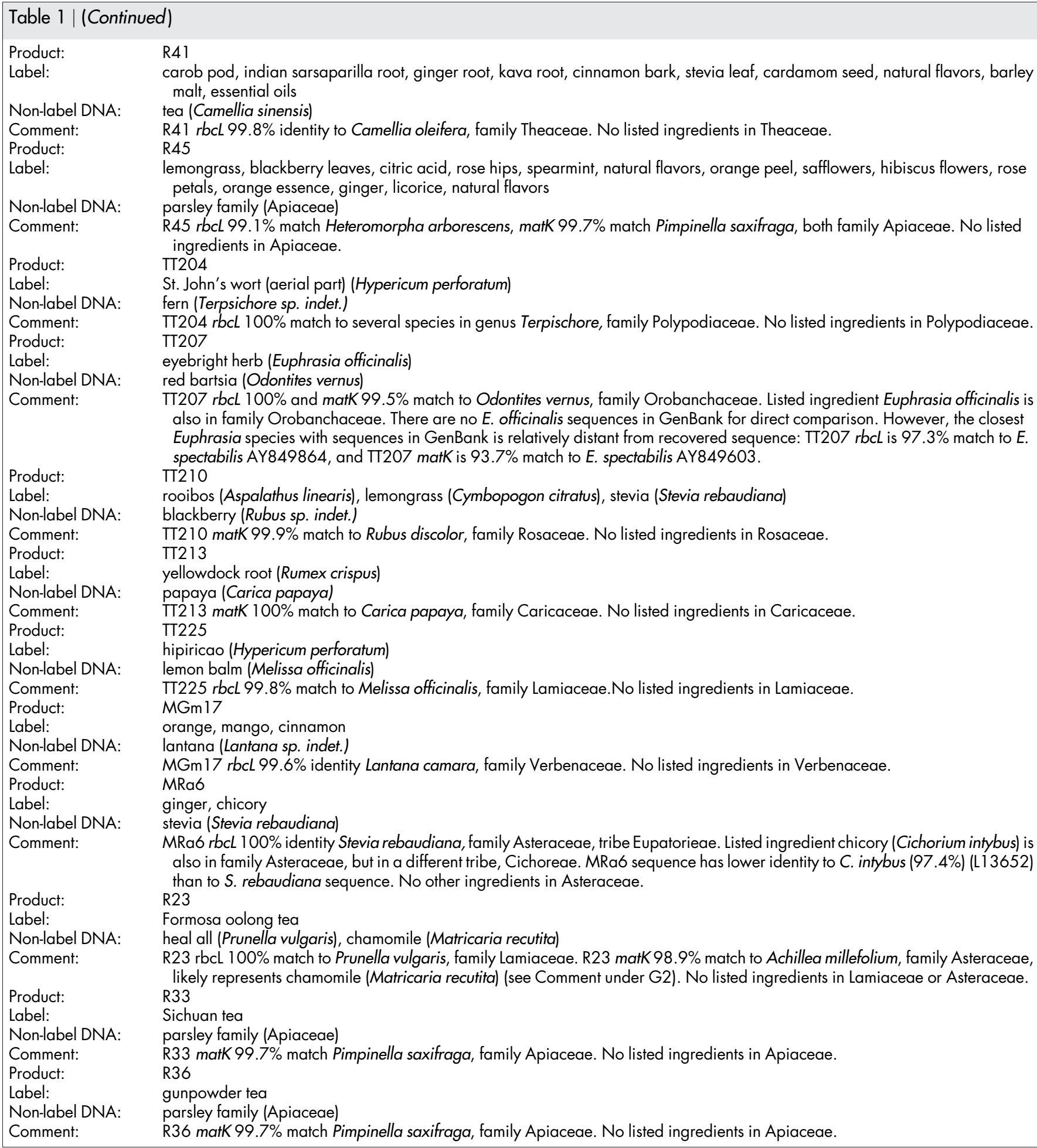

var. assamica vs. C. sinensis var. sinensis ( $71 \%$ vs. $12 \%, \mathrm{p}=0.0002)$ (additional details in Supplementary Table S3 online).

\section{Discussion}

Reliable DNA identification of species requires recovery of a barcode sequence from the sample, representation of relevant species in the reference database, and sufficient nucleotide sequence variability to distinguish among closely-related species $^{26}$. Regarding the first requirement, we recovered $r b c L$ or matK barcodes from $90 \%$ of commercial tea products using a single set of primers for each region. Success was less frequent with herbal as compared to CS teas (84\% vs 96\%), which may reflect primer mismatch, Taq inhibition, or DNA degradation in some of the diverse plant materials in herbal teas. In terms of markers, $r b c L$ was recovered from a broader taxonomic range of plants than matK ( 42 species in 24 families vs. 25 species in 16 families; Figs. 1,2). These results are consistent with general observation that $r b c L$ is more easily amplified from wide range of species than is mat $^{19,20}$.

The second condition for DNA identification of species is representation of relevant taxa in the reference database, in our case GenBank. As in most practical applications of barcoding, our specimens were morphologically unrecognizable, thus representation cannot be assessed directly. About one-third of the plant species listed on labels lacked GenBank records for $r b c L$, matK, or both at 
the time of the study. A more precise indicator of species representation is whether the recovered sequences are identical to any in the database. $62 \%$ of our barcode haplotypes did not have an identical match in GenBank (Figs. 1,2). This indicates that many plant species found in tea products are either not represented, have undocumented intraspecific variation, or that a sequencing error has occurred.

The third requirement for identifying species by barcode is biological: there must be sequence differences that discriminate among closely-related species. We can determine how well this condition is met for our specimens by comparing the best match and the congeneric nearest neighbor for each haplotype. For $r b c L$, these differed by only 1 site on average, and for matK these differed by only 2 sites on average (Figs. 1,2; see also Supplementary Tables S1,S2 online). Our results are consistent with the estimated $70 \%-85 \%$ species discrimination using $r b c L+$ matK barcodes, and highlight the relatively small number of positions that distinguish many closely-related plant species ${ }^{19,23,24}$. Differences between congeneric species in this study are similar to those reported for intraspecific variation and are also the same magnitude as sequencing error. Thus a barcode that differs from its closest reference database sequence at just one or a few sites plausibly represents an unrecorded variant for that species, a closely-related species not in the reference database, or sequencing error.

Our results highlight a need for improved algorithms for assigning taxonomic names to plant barcode sequences, particularly if barcoding is to be applied by non-specialists, which is one of the goals of the effort ${ }^{1,12,25}$. Algorithms that place search results in the context of plant taxonomy and current database representation of related plants will be helpful. Character-based approaches may assist in distinguishing closely-related species, particularly if supported by expert annotation that flags diagnostic nucleotide positions ${ }^{27,28}$. In addition, although employing two markers adds precision to plant barcode identifications, it also generates a need for algorithms that integrate database search results. In our data, most extractions that yielded both markers gave discordant results, that is, the $\mathrm{rbcL}$ and $m a t K$ barcodes matched different species in GenBank, largely reflecting differences in representation of species or intraspecific variants for the two markers.

A large fraction (35\%) of herbal products yielded one or more barcodes that pointed to non-label ingredients. Possible explanations include database errors (e.g. sequences with incorrect species names), limitations of search algorithm (e.g. relevant sequences not recognized by BLAST), laboratory error (e.g. PCR contamination, sample mix-up), or presence of unlisted ingredients. The disproportionate number of discordant sequences recovered from herbal specimens and the finding of species not listed on other products and not under study in the laboratory points to unnamed constituents. This could reflect inadvertent introduction, such as from harvested plant material mixed with unrecognized species, residual products in processing machinery, or as part of unspecified flavorings listed on some products. The relative amount of such potential material in our samples is unknown and is beyond the scope of this study. The finding of unlisted chamomile (M. recutita) or tea plant (C. sinensis) in multiple products suggests the possibility of addition or substitution to improve taste, appearance, or for economic reasons ${ }^{29}$.

To our knowledge, the polymorphism at $r b c L$ position 68 is the first described plastid marker that differs among C. sinensis varieties, regions of cultivation, and tea processing types ${ }^{5-11}$. Our results are consistent with marketplace trends-India and Sri Lanka, largely devoted to cultivation of $C$. sinensis var. assamica, are the dominant global exporters of black tea, whereas China, largely cultivating C. sinensis var. sinensis, has become the dominant exporter of green tea, with $75 \%$ of world market ${ }^{30}$. Our findings may help inform future research on the geographic origin and diversity of wild and cultivated CS resources ${ }^{5,31}$.
In summary, plant DNA barcodes can be recovered from most commercial tea products using a standard protocol. At the same time, interpreting DNA barcode identifications in relation to product labels is challenging. New algorithms that place search results in the context of standard plant names and character-based keys for distinguishing closely-related species are needed. With appropriate software to guide non-experts, DNA barcoding can offer an effective method to help provide more accurate ingredient labels to consumers, thereby improving safety of food and botanicals ${ }^{32}$. This is particularly pertinent in an increasingly global economy where longer and more complex market chains distance suppliers from the source of products and where regulatory agencies are becoming more stringent with food and botanical labeling ${ }^{33,34}$.

\section{Methods}

Specimen collection. CS and herbal tea products from New York City stores, school dining halls, and homes of investigators were collected during October 2009February 2010. 146 products were obtained from 25 locations, representing 33 manufacturers, 17 countries, and 82 plant common names. As this study was designed to enable comparison between CS and herbal teas and not among individual products or manufacturers, products were assigned an arbitrary alphanumeric code. 73 were C. sinensis, and 73 were herbal products prepared from other plant species. Five herbal products contained C. sinensis together with other plants. 44 herbal teas $(60.3 \%)$ listed a single ingredient; the remainder named 2-10 different plants. When not specified on the label, scientific and common name equivalents were determined from the reference used by the U.S. Food and Drug Administration ${ }^{35}$.

Reference samples. C. sinensis var. assamica specimens $(\mathrm{n}=17)$ were collected in Yunnan, China by SA during 2007-2009. C. sinensis var. sinensis specimens $(\mathrm{n}=24)$ collected in China (7), Taiwan (7), Japan (7), and Argentina (3) were obtained from the Kunming Institute of Botany, Kunming, China. Reference sample $r b c L$ sequences and additional collection information were deposited in GenBank under accession codes JN009623-JN009663. GenBank accessions used for comparison of C. sinensis rbcL haplotypes included C. albogigas (AF380033), C. granthamiana (AF380034), C. japonica (AF380035), C. oleifera (GQ436637), C. sasanqua (AF380036), C. sinensis (AF380037), and Tutcheria hirta (AF380067).

DNA extraction and sequencing. DNA was isolated from 5-15 mg dried tissue using a DNeasy96 Plant kit (Qiagen). The manufacturer's protocol was modified as follows: tissue was disrupted and then incubated for $12-18 \mathrm{~h}$ with gentle mixing at $42^{\circ} \mathrm{C}$ in $600 \mu \mathrm{L}$ of the supplied AP1 buffer with $600 \mu \mathrm{g}$ of protease $\mathrm{K}$ added $(630 \mu \mathrm{L}$ total volume). Polysaccharides were precipitated at $4{ }^{\circ} \mathrm{C}$ with $200 \mu \mathrm{L} \mathrm{AP} 2$. The remaining steps followed the manufacturer's protocol. For the $86 \%$ of specimens that appeared morphologically homogenous, a single extraction was performed. The remaining samples were divided into groups of morphologically homogeneous material (average 3 , range 2-8), and separate extractions were performed with the aim of recovering individual components.

Individual amplifications of mat $K$ and $r b c L$ took place in a $15 \mu \mathrm{L}$ volume containing: $1.5 \mu \mathrm{L}$ buffer [200 mM Tris $\mathrm{pH} 8.8,100 \mathrm{mM} \mathrm{KCl}, 100 \mathrm{mM}\left(\mathrm{NH}_{4}\right)_{2} \mathrm{SO}_{4}$,

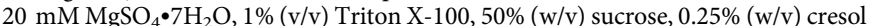
red], $0.2 \mathrm{mM}$ dNTPs, $0.025 \mu \mathrm{g} / \mu \mathrm{L}$ BSA, $0.5(r b c L)$ or 1 (matK) $\mu \mathrm{M}$ of each primer, 1 unit of Taq, and $0.5 \mu \mathrm{L}$ genomic DNA. For amplification and sequencing of $m a t K$, primers 3F (5'-CGT-ACA-GTA-CTT-TTG-TGT-TTA-CGA-G-3') and 1R ( $5^{\prime}$-ACC-CAG-TCC-ATC-TGG-AAA-TCT-TGG-TTC- $\left.3^{\prime}\right){ }^{27}$ were used with the following cycling conditions: $95^{\circ} \mathrm{C} 2.5 \mathrm{~min} ; 10$ cycles: $95^{\circ} \mathrm{C} 30 \mathrm{~s}, 56^{\circ} \mathrm{C} 30 \mathrm{~s}, 72^{\circ} \mathrm{C}$ $30 \mathrm{~s} ; 25$ cycles: $88^{\circ} \mathrm{C} 30 \mathrm{~s}, 56^{\circ} \mathrm{C} 30 \mathrm{~s}, 72^{\circ} \mathrm{C} 30 \mathrm{~s} ; 72^{\circ} \mathrm{C} 10 \mathrm{~min}$. For $r b c L$ amplification and sequencing, primers F1 (5' -ATG-TCA-CCA-CAA-ACA-GAG-ACT-AAA-GC$\left.3^{\prime}\right)^{22}$ and R634 (5'-GAA-ACG-GTC-TCT-CCA-ACG-CAT- $\left.3^{\prime}\right)^{20}$ were used with the following cycling conditions: $95^{\circ} \mathrm{C} 2.5 \mathrm{~min} ; 35$ cycles: $95^{\circ} \mathrm{C} 30 \mathrm{~s}, 58^{\circ} \mathrm{C} 30 \mathrm{~s}, 72^{\circ} \mathrm{C}$ $30 \mathrm{~s} ; 72^{\circ} \mathrm{C} 10 \mathrm{~min}$

PCR products were treated with ExoSAP-IT and bi-directionally sequenced with BigDye 3.1 chemistry on an ABI 3730 sequencer (High-Throughput Genomics Unit, University of Washington)

Portable laboratory. A subset of specimens (10) were analyzed in a portable laboratory. Equipment included a thermal cycler (Techne), microcentrifuge (Eppendorf minispin), vortex mixer, heating block, pipettemen, and E-gel apparatus (Invitrogen), purchased used or reconditioned except for E-gel unit. DNA was isolated with DNeasy Plant Mini Kit (Qiagen) following manufacturer's instructions. PCR was performed using $r b c L$ primers as described above except that $25 \mu$ reaction volume, 0.5 units TaKaRa Ex Taq, and buffer supplied by manufacturer were used. DNA and PCR yields were assessed on an E-gel EX 1\% with a blue-light excitable nucleic acid stain, products were cleaned with QIA quick PCR purification kit (Qiagen), and unidirectional sequencing was performed at a commercial facility (Macrogen).

Sequence files and data analysis. Trace files were assembled in MacVector 11.0, and sequences with greater than $2 \%$ ambiguous bases were discarded, using QV of 40 for 
bi-directional reads and 20 for single reads. Sequences were aligned using ClustalW $(r b c L)$ or MUSCLE v3.8.31 (matK). Sequence files are deposited in GenBank under accession codes HQ699082-HQ699129 ( $r b c L$ ) and HQ699130-HQ699169 (matK). Fisher's exact test, two-tailed, was used for statistical comparisons.

Database searches. GenBank database was searched using megaBLAST during August-October 2010, with default parameters adjusted to retrieve 5000 sequences. To optimize correct identifications, the closest match for each $r b c L$ and matK haplotype was defined as the target with highest percentage identity using an arbitrary cutoff of $90 \%$ or greater overlap with the query sequence. In most cases this corresponded to the sequence with the highest BLAST score. In other cases, the closest match was a shorter target with a higher percent identity. Ambiguous bases in query or target sequences were considered as matching. For queries that produced multiple identical matches, the target with a species name closest to a label ingredient was chosen when possible. A similar procedure was followed for BOLD searches, with the exception that the number of alignment results was 100 , which is the maximum allowed. For consistency in reporting, the species of sequences deposited in GenBank and BOLD were used unaltered even though some may be in error or reflect outdated taxonomy.

1. Hebert, P. D. N., Cywinska, A., Ball, S. L. \& deWaard, J. R. Biological identifications through DNA barcodes. Proc. Biol. Sci., 270, 313-321 (2003).

2. Chang, H. T. A taxonomy of the genus Camellia. Acta Sci. National Uni.v Sunyatseni, Monog. Series 1, 1-180 (1981).

3. Chang, H. T. \& Bartholomew, A. Camellias. London: Batsford, 211 p. (1984).

4. Ming, T. \& Zhang, W. The evolution and distribution of genus Camellia. Acta Botanica Yunnanica 18, 1-13 (1996).

5. Balasaravanan, T., Pius, P. K., Kumar, R. R., Muraleedharan, N. \& Shasany, A. K. Genetic diversity among south Indian tea germplasm (Camellia sinensis, $C$. assamica, and C. assamica spp. Lasiocalyx) using AFLP markers. Plant Sci. 165, 365-372 (2003).

6. Ni, S., Yao, M., Chen, L., Zhao, L. \& Wang, X. Germplasm and breeding research of tea plant based on DNA marker approaches. Front. Agric. China 2, 200-207 (2008).

7. Katoh, Y., Katoh, M., Takeda, Y. \& Omori, M. (2003) Genetic diversity within cultivated teas based on nucleotide sequence comparison of ribosomal RNA maturase in plastid DNA. Euphytica 134, 287-295 (2003).

8. Wachira, F. R., Powell, W. \& Waugh, R. An assessment of genetic diversity among Camellia sinensis L. (cultivated tea) and its wild relatives based on randomly amplified polymorphic DNA and organelle-specific STS. Heredity 78, 603-611 (1997).

9. Chen, J., Wang, P., Xia, Y., Xu, M. \& Pei, S. Genetic diversity and differentiation of Camellia sinensis L. (cultivated tea) and its wild relatives in Yunnan province of China, revealed by morphology, biochemistry, and alloenzyme studies. Genet. Resources Crop. Eval. 52, 41-52 (2005).

10. Singh, D. \& Ahuja, P. S. 5S rDNA gene diversity in tea (Camellia sinensis (L.) O. Kuntze) and its use for variety identification. Genome 49, 91-96 (2006).

11. Lai, J.-A., Yang, W.-C. \& Hsia, J.-Y. An assessment of genetic relationships in cultivated tea clones and native wild tea in Taiwan using RAPD and ISSR markers. Bot. Bull. Acad. Sin. 42, 93-100 (2001).

12. Li, H. L. The domestication of plants in China: ecogeographical considerations. In: Keightley, D. N., editor. The Origins of Chinese Civilization. Berkeley: University of California Press, pp. 21-64 (1982).

13. Ceresa, M. (1996) Diffusion of tea-drinking habit in pre-Tang and early Tang period. Asiatica Venetiana 1, 19-25 (1996).

14. Magoma, G. N., Wachira, F. N., Obanda, M., Imbuga, M. \& Agong, S. G. The use of catechins as biochemical markers in diversity studies of tea (Camellia sinensis). Genet. Resources Crops. Evol. 47, 107-114 (2000).

15. Ahmed, S., et al. Pu-erh tea tasting in Yunnan, China: correlation of drinkers' perceptions to phytochemistry. J. Ethnopharmacol. 132, 176-185 (2010).

16. Centers for Disease Control. Anticholinergic poisoning associated with an herbal tea-New York City, 1994. Morb. Mortal. Wkly. Rep. 44, 193-195 (1995).

17. Kumana, C. R. et al. Herbal tea induced veno-occlusive disease: quantification of toxic alkaloid exposure in adults. Gut 26, 101-104 (1985).

18. Toxicology and Clinical Pharmacology of Herbal Products, editor Cupp, M. J. Totowa: Humana Press, 325 p. (2000).
19. CBOL Plant Working Group. A DNA barcode for land plants. Proc. Natl. Acad. Sci. U. S. A. 106, 12794-12797 (2009).

20. Fazekas, A. J. et al. Multiple multilocus DNA barcodes from the plastid genome discriminate plant species equally well. PLoS ONE 7, e2802 (2008).

21. Seberg, O. \& Petersen, G. How many loci does it take to barcode a crocus? PLoS ONE 4, e4598 (2009).

22. Kress, W. J. \& Erickson, D. L. A two-locus global DNA barcode for land plants: the coding $r b c L$ gene complements the non-coding trnH-psbA spacer region. PLoS ONE 2, e508 (2007).

23. Fazekas, A. J. et al. Are plant species inherently harder to discriminate than animal species using DNA barcoding markers? Mol. Ecol. Res. 9(S1), 130-139 (2009).

24. Lahaye, R. et al. DNA barcoding the floras of biodiversity hotspots. Proc. Natl. Acad. Sci. U. S. A. 105, 2923-2938 (2008).

25. Chase, M. W. et al. Land plants and DNA barcodes: short-term and long-term goals. Phil. Trans. R. Soc. B 360, 1889-1895 (2005).

26. Hebert, P. D. N., Stoeckle, M. Y., Zemlak, T. S. \& Francis, C. M. Identification of birds through DNA barcodes. PLoS Biol. 2, e312 (2004).

27. Little, D. P. \& Stevenson, D. W. A comparison of algorithms for the identification of specimens using DNA barcodes: examples from gymnosperms. Cladistics 23, 1-21 (2007).

28. Hollingsworth, P. M., Graham, S. W. \& Little, D. P. Choosing and using a plant DNA barcode. PLOS ONE 6, e19254.

29. Cole, M. R. \& Fetrow, C. W. Adulteration of dietary supplements. Amer. J. HealthSystem Pharm. 60, 1576-1580 (2003)

30. Food and Agricultural Organization of the United Nations. Medium-term prospects for agricultural commodities. Projections to the year 2010. Rome, 2010. Accessed online at http://www.fao.org/docrep/006/y5143e/y5143e0z.htm

31. Lou, S. K., Wong, K. L., Li, M., But, P. P., Tsui, S. K. \& Shaw, P. C. An integrated web medicinal materials DNA database: MMDBD (Medicinal Materials DNA Barcode Database). BMC Genomics 11, 402 (2010).

32. Prince, L. M. \& Parks, C. R. Phylogenetic relationships of Teaceae inferred from chloroplast DNA sequence data. Amer. J. Botany 88, 2309-2320 (2001).

33. Ebeler, S. E., Takeoda, G. R. \& Winterhalter, P., editors. Authentication of Food and Wine. United States of America: Oxford University Press, 364 p. (2007).

34. Chen, S.et al. Validation of the ITS region as a novel DNA barcode for identifying medicinal plant species. PLoS ONE 5, e6813 (2010).

35. McGuffin, M., Kartesz, J. T., Leung, A. Y. \& Tucker, A. O., editors. Herbs of Commerce, $2^{\text {nd }}$ Edition. United States of America: American Herbal Products Association, 421 p. (2000).

\section{Acknowledgments}

We thank Jesse Ausubel (JA) for encouraging investigation, Trinity School for permission to work with their students, and JA and Paul Waggoner for helpful comments on an earlier version of the manuscript. DPL was supported by a grant from The Alfred P. Sloan Foundation (2010-06-02). SA's field work in Yunnan was supported by NSF grants DDEP OISE-0749961 and NSF EAPSI OISE-0714431. SA thanks the Kunming Institute of Botany, Chinese Academy of Sciences for permission to collect CS samples.

\section{Author contributions}

MYS and DPL designed the study; SA, CCG, RK, and GY contributed samples; MYS, CCG, RK, GY, and DPL performed experiments and analyzed data; and MYS and DPL wrote the manuscript with assistance from all authors.

\section{Additional information}

Supplementary Information accompanies this paper at http://www.nature.com/ scientificreports

Competing financial interests: The authors declare no competing financial interests.

License: This work is licensed under a Creative Commons

Attribution-NonCommercial-ShareAlike 3.0 Unported License. To view a copy of this license, visit http://creativecommons.org/licenses/by-nc-sa/3.0/

How to cite this article: Stoeckle, M.Y. et al. Commercial Teas Highlight Plant DNA Barcode Identification Successes and Obstacles. Sci. Rep. 1, 42; DOI:10.1038/srep00042 (2011) 